\title{
Tension pneumothorax during foreign body retrieval: experiences with 2 cases
}

Xu Zhang, Jinhong Wu, Yuexing Zhu, Weixing Li \& Wenxian Li

Department of Anesthesiology, The Eye, Ear, Nose and Throat Hospital, Fudan University, Shanghai, China

\section{Background}

Tension pneumothorax during surgery may cause severe hypotension and immediate circulation collapse, leading to a fatal emergence unless treated by decompression. Foreign body aspiration is a leading cause of accidental death among preschool children [1]. We report two cases, both developed rapid onset of tension pneumothorax during a rigid bronchoscope procedure, underwent thoracentesis and were discharged without cerebral hypoxia sequelae. We also discuss the probable etiologies and present our therapy experience

\section{Case Report}

Case

An 8-month old girl, weighing $9.5 \mathrm{~kg}$, with suspected aspiration of peanut for one day Physical examination showed: decreased breathing in left lung and a rhonchi sound in right lung. The patient presented without cyanosis or breathlessness. Anesthesia was induced with $8 \%$ sevoflurane. After establishing intravenous access, a bolus of propofol $50 \mathrm{mg}$, fentanyl $20 \mu \mathrm{g}$, methylprednisolone $20 \mathrm{mg}$, dexmedetomidine $4 \mu \mathrm{g}$ and mivacurium chloramine $2 \mathrm{mg}$ were administered. Maintenance of anesthesia was facilitated by a continuous infusion of propofol $100 \mathrm{mg} / \mathrm{h}$ and a bolus of muscle relaxant $(1 \mathrm{mg}$ mivacurium). After topical anesthesia with $2 \%$ lidocaine $(2 \mathrm{ml})$ in epiglottic vallecula and subglottic region, the surgeon inserted the rigid bronchoscopy. The ventilation was achieved using a manually controlled jet ventilator (Manujet III, VBM,Germany). After 8 minutes a large fragment of peanut in the main tracheal was retrieved. The procedure ended 15 minutes later. After extraction of the rigid bronchoscope, ventilation was maintained with jet ventilation of $100 \%$ oxygen, but the $\mathrm{SPO}_{2}$ decreased to $86 \%$. An endotracheal tube was inserted immediately and IPPV ventilation was used, but the deoxygenation was sustained with the $\mathrm{SPO}_{2}$ fluctuating from $86 \% \sim 90 \%$. A bedside ches ray was immediately taken and showed: Right pneumothorax, with lung collapse and shift of the mediastinum to the left (arrow), indicating that there was a tension pneumothorax (Fig 1). A thoracic surgeon was called. During this time, the arterial blood gas analysis shows: $\mathrm{PH} 7.30, \mathrm{PaO}_{2} 87 \mathrm{mmHg}$ and $\mathrm{PaCO}_{2} 40 \mathrm{mmHg}$. The anesthetic was terminated. When the patient had steady and sufficient tidal volume and purposeful movement, the tracheal tube was removed. The patient maintained $\mathrm{SPO}_{2}>93 \%$ through a nasal cannula of $2 \mathrm{~L} / \mathrm{min}$ flow oxygen. A right pleural cavity drainage was inserted afte anesthesia was established with sevoflurane $(4 \%)$, propofol $(40 \mathrm{mg})$ and insertion of a $2 \#$ classic laryngeal mask airway. The patient was extubated without further complication. The X-ray on the third day postoperatively showed the pneumothorax vanished, and the chest tube was removed 5 days later. The patient was discharged without any complications.

\section{Case 2}

A two-year-old boy, weighing $8 \mathrm{~kg}$, was admitted to our emergency department with a suspected foreign body (peanut) inhalation for more than 10 days. Preoperative examination showed reduced auscultation of right lung and the chest CT described: emphysema in the right bronchus with suspicious foreign body (Fig 2). The $\mathrm{SPO}_{2}$ was $100 \%$ preoperatively. Anesthesia was induced with $8 \%$ sevoflurane inhalation. After establishing intravenous access, a bolus of propofol $30 \mathrm{mg}$, fentanyl $10 \mu \mathrm{g}$, methylprednisolone $15 \mathrm{mg}$, succicholyne $20 \mathrm{mg}$ was administered. A spray of of $2 \%$ lidocaine of $1.5 \mathrm{ml}$ was used for airway topical anesthesia. Anesthesia was maintained with an infusion of propofol $200 \mathrm{mg} / \mathrm{h}$. Manual jet ventilation was used during the procedure (pressure below 10psi, frequency 15). After 5 minutes the surgeon retrieved part of the foreign body. The 3.5 rigid bronchoscope was inserted into the tracheal for the second time and the chest inflations diminished under the jet ventilation. The $\mathrm{SPO}_{2}$ decreased to $90 \%$. The chest cavities were overexpansed,and followed by circulatory

compromise $(\mathrm{HR}<60 \mathrm{bpm})$. The surgery was terminated and the rigid bronchoscopy was withdrawn. Successful tracheal intubation was done on the first trial, but the tongue was swollen and the mandible was stiff. A suspected tension pneumothorax was suspected Positive pressure ventilation was insufficient and the $\mathrm{SPO}_{2}$ dropped to $30 \%$. Rapid chest decompression was done, combined with a bolus of adrenaline $10 \mu \mathrm{g}$ (twice with 2 minutes interval). Immediate thoracentesis was done by anesthesiologist, with 14 gauge needle in the 2nd intercostal space in the midclavicular line. A"hiss" of rapid exit of air was heard immediately. The $\mathrm{SPO}_{2}$ increased to $85 \%$ and $\mathrm{HR}$ to $140 \mathrm{bpm}$. Dexmedetomidine $(0.6 \mu \mathrm{g}$ $\mathrm{kg} / \mathrm{min}$ ) was added for supported ventilation therapy. A bedside chest X-ray showed (Fig 3 ): Bilateral Tension pneumothorax (right lung compressed $30 \%$, left lung $60 \%$ ). After insertion of thoracostomy tubes on both sides, the patient improved gradually. The chest radiography on the next day demonstrated evolving right pulmonary infiltrates. The patient was discharged 3 weeks later for anti-inflammatory therapy without cerebral hypoxic complications.
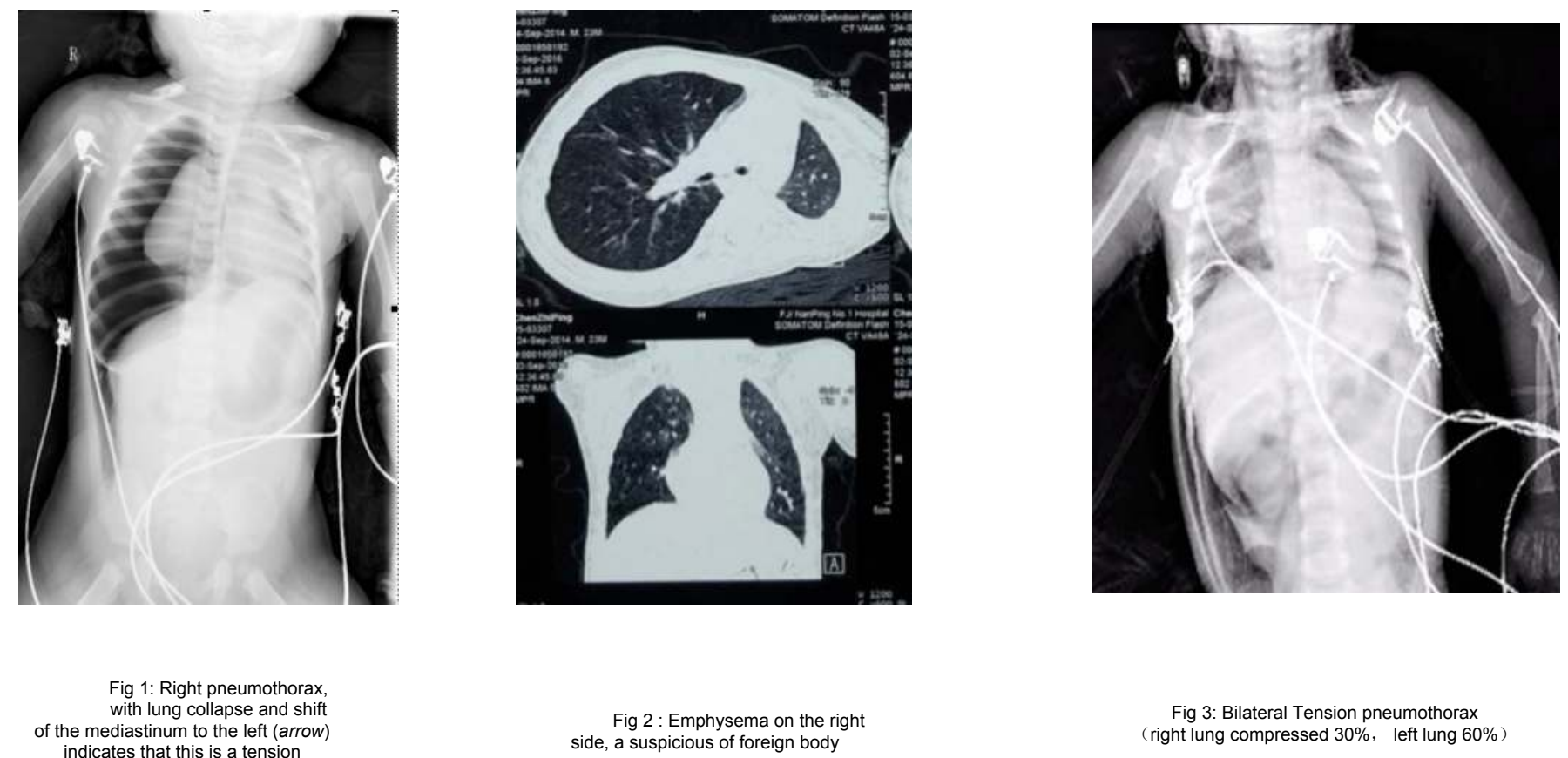

\section{Discussion:}

Pneumothorax is described as a large volume of air that collects and compresses the lung and heart. In an emergency situation, a chest tube is used to remove the trapped air. Pneuomthorax can occur in two conditions: lung leakage and positive ventilation. Aspiration of organic substances (such as nuts) usually creates intense inflammatory response. The cause of damage may arise from an underlying lung disorder, such as pneumonia (usually with empyema), abnormal pathological change, iatrogenic laceration or high positive pressure through surgery, and lung injury with increased inflammation. Tension pneumothorax may cause an acute episode of hemodynamic collapse (hypotension and cardiac arrest) and deterioratation of oxygenation (severe hypoxemia), usually caused by a collapsed lung, seldom occurring on both sides [2] Manual jet ventilation (MJV, Manujet III, VBM,Germany) was used in both cases. It was performed before insertion of the bronchoscopy by placing a fine tube (inner diameter= $0.5 \mathrm{~mm}$, we use $8 \mathrm{Fr}$ suction tube) transnasally into the trachea (about $2 \mathrm{~cm}$ at subglottic region). MJV can provide quick and efficient oxygenation during the procedure. MJV has a high-pressure oxygen/air outlet, which is adjustable usually below 20 psi.

In these two cases, there are some specific issues that should be reviewed:

1. The anesthetic plan:

Though there's still lack of guidelines of anesthetic management in FB surgery, we prefer to apply the flowchart of anesthetic protocol in our anesthesiology departmen at the Eye \& ENT Hospital of Fudan university (Fig 4). The associated ventilation method during rigid bronchoscopy includes: assistant positive ventilation, manually jet ventilation and spontaneous ventilation. The choice of ventilation strategy depends upon the clinical situation and anesthetist's experience.

When ventilation is ineffective during the surgery, there will be an faulty practice to blindly increase the airway pressure or ventilation frequency. The following

considerations should be take into account:

Misplacement of the jet ventilation tube (obstruction by instrument or shift the position to bronchial/oropharynx region)

Insufficient anesthetic depth (the usually cause of laryngospasm/bronchospasm, an increase dose of the anesthesia should be consider if intense airway

stimulation persists)

If no improvement happens, changing the ventilation mode (from jet ventilation to assistant mechanical ventilation or spontaneous ventilation)

While in jet ventilation, the anesthesiologist should focus on: anesthesia monitor anesthesia infusion, air pressure of jet ventilator, movement of thorax, surgical. To prevent iatrogenic injury, the following precautions should be taken to decrease possible barotrauma:

Focus on the jet ventilation parameters: lockout pressure, pressure (usually below 20 psi) and the frequency

Fix attention on patient's chest wall motion (efficient inflation and deflation periods according manually respiration rate, especially in expiratory phase, combined by auscultation)

Avoid vocal cord closure (especially after withdraw of rigid bronchoscopy)

2. Communication with surgeon:

The communication must take place during the whole peri-operatively period. It starts before the surgery, including the surgery type, the situation of the patient, the anesthetic plan and possible complications. During the surgery, the communication referred to the agreement of accordingly anesthetic plan and the surgical progress, which gives vital information for the anesthesiologist to pay attention to when planning the anesthetic, especially when airway stimulation is increased (the key to reducing adverse events). If the patient's status deteriorats, what precautions should be taken?

3. Decrease the pneumothorax related mortality and morbidity:

In tension pneumothorax, cardiovascular collapse and sustained hypoxemia indicate further compression of the lung [3], a prompt decompression should be considered as soon as possible, even before a radiological confirmation, which may be unavailable sometimes. The diagnosis of tension pneumothorax can be confirmed by hiss of air on decompression as in the second case. In the previous study, the tension pneumothorax can occur in $5.4 \%$ of major trauma patients, $64 \%$ of them were ventilated [4]. The needle decompression site should be in the $2 \mathrm{nd} / 3 \mathrm{rd}$ intercostal space (ICS), mid-clavicular line (MCL), and may be safer at 4th or 5th ICS in the midaxillary line [5]. The tube thoracostomy should be performed by skilled hands to decrease related complications.

4. The benefit of spontaneous ventilation: In patient with airway injury, especially with high positive thorax pressure, spontaneous breathing modes may help decrease ventilator-induced lung injury[6], improve pulmonary ventilation/perfusion (V/Q) matching and increased oxygenation[7]. The $a_{2}$ agonist dexmedetomidine could attenuate airway-circulatory reflexes [8], maintain adequate sedation without hemodynamic instability or respiratory-drive depression[9], may improve the final outcome of the pneumothorax.

Thoracic drainage is the major therapeutic method for thorax pneumothorax, the procedure should be done by an experienced hand to decrease complications [10] In an emergency situation (ie, circulation collapse) a prompt thoracocentesis must be done. The surgery team with a good communication should understand the pathophysiologic changes of foreign body aspiration, identify the high risk of airway injury and make early detection of pneumothorax, establish a deliberated anesthetic plan, and reduce the potential complications and improve the outcome. 